\title{
Prevalence of Hepatitis B Surface Antigen Among Students of Medical Laboratory Sciences in Port Sudan Ahlia College
}

\author{
Abd Elrahman Mustafa Abd Elrahman Osman, Shingray Osman Hashim, \\ Mohammed Abdall Musa, Omer Mohammed Tahir
}

Medical Laboratory Sciences Division, Port Sudan Ahlia College, Port Sudan, Sudan

Email address:

abdo_rahman96@hotmail.com (A. E. M. A. Osman)

To cite this article:

Abd Elrahman Mustafa Abd Elrahman Osman, Shingray Osman Hashim, Mohammed Abdall Musa, Omer Mohammed Tahir. Prevalence of Hepatitis B Surface Antigen Among Students of Medical Laboratory Sciences in Port Sudan Ahlia College. International Journal of HIV/AIDS Prevention, Education and Behavioural Science. Vol. 4, No. 1, 2018, pp. 1-4. doi: 10.11648/j.ijhpebs.20180401.11

Received: October 11, 2017; Accepted: December 15, 2017; Published: February 7, 2018

\begin{abstract}
Hepatitis B virus infection is a salient occupational hazard for health workers. It has been estimated that about two billion people worldwide have been infected with the virus. It is the $10^{\text {th }}$ leading cause of death worldwide and results in 500,000 to 1.2 million deaths per year due to cirrhosis and hepatocellular carcinoma. The presence of HBsAg in serum or plasma is an indication of active Hepatitis B infection, either acute or chronic. Healthcare workers, of which medical students are a part of, are at high risk of encountering accidental needle prick injuries, blood and body fluid exposure and hence acquiring blood borne infections, especially Hepatitis B which may be followed by serious long term sequelae in a significant number of cases. The aim of this study was to determine the seroprevalence rate of Hepatitis B surface antigen $(\mathrm{HBs} A g)$ among the Port Sudan Ahlia College, Department of Medical Laboratory Sciences students, during the period from January to May 2012 AD. The study included male and female individual's aged18-25 years. It was found that the incidence of hepatitis B virus among the students under study amounted to $2 \%$ using the Immuno-Chromatography Test (ICT). Seroprevalence was recorded in the females $(2.0 \%)$ than males $(0.0 \%)$ students. Other studies are required in the future for more confirmation with more specific and sensitive Techniques; such as: analysis by Enzyme linked Immuno Sorbent Assay (E. L. I. S. A.) Technique and Polymerase Chain Reaction (P. C. R). Participated in this study, qualitative detection of HBsAg was done using one step HBsAg rapid test strips (DiaSpot Diagnostics, USA). Results were correlated and reported as positive or negative. Among the 50 samples analyzed, 2 samples were positive for HBsAg accounting for a prevalence rate of $2.0 \%$. This prevalence was however statistically non - significant ( $\mathrm{p} 0.05$ ). The major risk factors of hepatitis B transmission among medical students include unprotected exposure body fluids, blood and blood products as well as lack of vaccination.
\end{abstract}

Keywords: Seroprevalence, Port Sudan, Hepatitis B Virus, ICT, E. L. I. S. A., P. C. R.

\section{Introduction}

Hepatitis B is a salient occupational hazard for health workers. It is a viral infection that attacks the liver and can cause both acute and chronic disease. It has been estimated that about two billion people worldwide have been infected with the virus, 350million people worldwide are chronic Hepatitis B virus (HBV) carriers, and about

600,000 people die every year as a result of the consequences of Hepatitis B [1, 2].

Hepatitis $B$ virus is the 10th leading cause of death worldwide and results in 500,000 to 1.2 million deaths per year due to cirrhosis and hepatocellular carcinoma [3]. HBV is transmitted through contact with the blood or other body fluids of an infected person. The route of HBV transmission is similar to that of HIV transmission. However, HBV is 50 to 100 times more infectious than HIV and 10 times more than Hepatitis C virus [2, 4]. Many of the patients infected with $\mathrm{HBV}$ are asymptomatic and as such helpin the spread of the disease in the community and particularly to health workers [5]. HBV also has a perinatal route of transmission (from mother to child at birth) this route is a common source 
of transmission in Asia and Africa [6, 7].

The presence of HBsAg in serum or plasma is an indication of active Hepatitis $\mathrm{B}$ infection, either acute or chronic. HBV is the most common significant chronic viral infection worldwide. The endemicity of HBV infection varies greatly worldwide. The prevalence of chronic HBV infection varies from $\geq 8 \%$ in Africa, Asia, and Western Pacific to $<2 \%$ in Western Europe, North America and Australia [7]. In Nigeria where HBV infection is considered endemic, different prevalence rates have been reported. The several reports have established the endemic nature of HBV by the presence of HBsAg in different population groups from different parts of the country.

A HBsAg Seroprevalence rate of $14.3 \%$ was reported in Jos, Nigeria [9], 4.3\% prevalence rate was reported in pregnant women in Port Harcourt, Nigeria [10]. Among blood donors in Benin City, a prevalence of $5.4 \%$ was reported [11], while a prevalence rate of $25.0 \%$ was reported among HIV infected individuals in Jos, Nigeria [9]. The incubation period of HBV as defined by the duration between exposure and appearance of clinical jaundice is between 30 and 130 days. The serologic incubation period, the duration between exposure and appearance of Hepatitis B surface antigen in the plasma or serum as detected by radioimmunoassay is as early as 6days and rarely persists for more than 4months $[12,13]$. The HBsAg one step Hepatitis B surface antigen test strip (serum/plasma) is a rapid test to qualitatively detect the presence of HBsAg in serum or plasma sample. The test employs a combination of monoclonal and polyclonal antibodies to selectively detect elevated levels of HBsAg in serum or plasma. Health care workers (HCWs) are those persons such as employees, students, contractors, attending clinicians, public safety workers or volunteers whose activities involve contact with patients or with blood or other body fluids from patients in a healthcare laboratory or public setting. HCWs are at high risk of encountering accidental needle prick injuries, blood and body fluid exposure and hence acquiring blood borne infections, especially Hepatitis B and C, which may be followed by serious long term sequelae in a significant number of cases $[14,15]$.

The aim of this study was to determine the seroprevalence rate of Hepatitis $\mathrm{B}$ surface antigen (HBsAg) among the medical Laboratory students of Port Sudan Ahlia College.

\section{Materials and Methods}

\subsection{Ethical Consideration}

Ethical clearance was sought for and obtained from the ethical committee of Port Sudan Ahlia Colllege. Informed consent was also obtained from all the participants.

\subsection{Study Population}

Medical students (Medical Laboratory Science) of Port Sudan Ahlia College. were recruited for this study. A total of fifty (50) of the students participated. Simple random sampling technique was used in recruiting the subjects of this study.

\subsection{Exclusion Criteria}

Students who had received Hepatitis B vaccine were excluded from the study. Sociodemographic data and other relevant information of each participant were obtained with the aid of a questionnaire.

\subsection{Specimen Collection}

$3 \mathrm{ml}$ of venous blood samples were aseptically collected from the cubital fossa of all the participants and dispensed into sterile plain containers. The samples were allowed to stand on the bench for 15 minutes for clotting and clot retraction. Subsequently, the samples were centrifuged at $4000 \mathrm{rpm}$ for 5 minutes. The serum was separated into prelabeled plain containers.

\subsection{Sample Analysis}

Qualitative detection of serum HBsAg was determined using commercial third generation rapid chromatographic immunoassay. One commercial rapid diagnostic HBsAg test strip were used; HBsAg rapid test strip (DiaSpot Diagnostics, USA) with relative sensitivity and specificity of $99 \%$ and $97 \%$ respectively with an accuracy of $98.5 \%$. Strict adherence to manufacturers' instructions was observed.

The test and result interpretations were done according to the manufacturers' instructions. Result of the test strip were correlated for each subject and reported as positive or negative.

\subsection{Data Analysis}

Data was analyzed using Chitest, p value of 0.05 was considered significant at $95 \%$ confidence interval Data analysis was done with the aid of Statistical Programme for Social Sciences (SPSS) version 20.0.

\section{Results}

A total of 50 individuals randomly selected in Ahlia Collage, Faculty of Medical Laboratory Sciences for students were enrolled in this study.

The state of 50 serum samples were tested for hepatitis B surface antigen, Showing (2\% were positive using rapid test strip (DiaSpot Diagnostics, USA). While (98\%) were negative using the same method.

Table 1. Classification of classes according to number of positive and negative results.

\begin{tabular}{lll}
\hline Classes NO. & HBs Ag positive & HBs Ag negative \\
\hline Class 1 & 1 & 9 \\
Class 2 & 0 & 15 \\
Class 3 & 0 & 10 \\
Class 4 & 0 & 15 \\
\hline
\end{tabular}




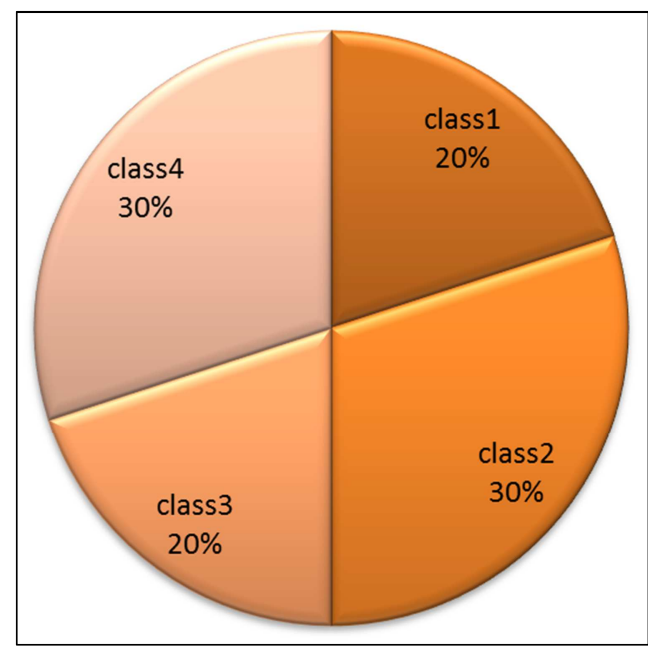

Figure 1. Percentage of students according to class.



Figure 2. Percentage of results

\section{Discussion}

Hepatitis B virus (HBV) remains a public health burden in the world, mostly in developing countries including Sudan. Occupational exposure of healthcare workers to infected blood and blood products remain a major risk factor. Medical students are at risk of acquiring HBV infection and as well transmitting it to other patients in the course of their clinical and laboratory posting.

HBV infections are common serious infection. The current study presented the prevalence of HBV infection among students of medical laboratory sciences faculty of Port Sudan Ahlia Collage to demonstrate the infections within them in both gender. The students a rounded 18-25 years during the four classes Conceding the data analysis the result appear the subject groups in target Collage negative except one student in class one, either rapid test (ICT)of HBs Ag. Last result must be confirmed by ELISA or PCR or other technique. The outcome of study by statistical method 2\% targets are positive in places, $98 \%$ are negative. These results indicated the high healthy improvement around the student in young age. This may be due to their behavior, traditional and may due to the efforts taken by families about importance of vaccination. Unfortunately no recent studies among students in Faculty of Medical Laboratory Sciences are available. So, the data obtained from this study did not compared with other previous studies.

The prevalence of HBV has been shown by previous studies to vary between developed countries where the prevalence is low $(2 \%)$ and developing countries where the infection is endemic with age, sex, and socioeconomic status as important risk factors for infection [16].

This study showed HBsAg Seroprevalence rate of $2.0 \%$ among medical Laboratory students of Port Sudan Ahlia College. This low prevalence rate is comparable to the prevalence rate of $39 \%$ reported by Olubuyida and colleagues among doctors and dentists [17], but in contrast to the prevalence rate of $3.2 \%$ reported among medical students of Lagos State University [18].

Results from other parts of Africa and the rest of the world show varying result, Pido and Kagimu reported a prevalence of $11.0 \%$ among medical students of Maker ere University, Uganda [19], prevalence of $0.6 \%$ and $0.95 \%$ were reported among Gomal University medical students, Pakistan [20] and students of health colleges in Saudi Arabia [21] respectively. However, study at Kenyatta National Hospital reported a high prevalence of $18 \%$ among the medical students [22]. The prevalence rate was higher in females $2 \%$ (1.0 0f 50) than in males $0.0 \%$ ( 0.0 of 50$)$ this relative disparity in the prevalence rates between males and females is incomparable to the study by Dutta and colleagues which reported HBsAg prevalence of $35.3 \%$ in males and $19.3 \%$ in females [24]. No scientific evidence has been given for this apparently higher Seroprevalence in males than in females.

In this study, HBsAg was highest among the 21-24years age group. This is comparable to the study by Eke and colleagues which found HBsAg to be highest among women of 20-24years age group in Nigeria [23]. In most epidemiological studies on $\mathrm{HBsAg}$, there has been a link between age and acquisition of HBsAg [24]. The age of acquiring the infection is one of the determinants of the prevalence rates of $\mathrm{HBsAg}$.

\section{Conclusion and Recommendations}

\subsection{Conclusion}

In this small size study among the student in Port Sudan Ahlia Collage, department of Medical Laboratory Sciences, the prevalence of HBs Ag (positive results) represents (2\%). While the negative results represented (98\%) by using screening techniques (ICT). the result obtained could possibly reflect the prevalence of HBs Ag infection in faculty of Ahlia Collage department of Medical Laboratory Sciences. Lack of vaccination and unprotected exposure to body fluids, blood and blood products remains the major risk of Hepatitis $\mathrm{B}$ transmission among medical students.

\subsection{Recommendations}

I. Effective health planning strategies are required to increase the public awareness of infection with viral diseases.

ii. Urgent further good quality epidemiological studies are 
needed.

iii. Wide spread educational and vaccination programs between the students are recommended.

iv. Further in depth studies using large sample size at different techniques, and employing recent molecular characterization techniques (e.g.: Real Time Polymerase Chain Reaction R. T. P. C. R) are recommended.

$\mathrm{v}$. We recommend that medical students be duly informed and vaccinated against $\mathrm{HBV}$ and given proper orientation on the various routes of transmission of the virus after voluntary HBsAg screening. Safety guidelines should be enforced in the hospital and those who have acquired the infection be treated to prevent horizontal transmission to their colleagues and patients.

\section{References}

[1] Maddrey W. C. Clin. Lab, 2001, 47 (1-2), 51-55.

[2] WHO. Hepatitis B factsheet. World Health Organisation, Geneva, Switzerland. 2012. Available from http://www.who.int/mediacentre/factsheet/fs204/en/ retrievd 27/02/2013

[3] Levanchy D. Journal of Viral Hepatology, 200411, 97-107.

[4] Center for Disease Control and Prevention (CDC). Updated U.S public health service guidelines for the management of occupational exposure to $\mathrm{HBV}, \mathrm{HCV}$ and $\mathrm{HIV}$ and recommendation for post exposure prophylaxis. CDC MMWR, 2011, 50 (RR11), 1-42.

[5] Choudharry I. A. and Khan S. A. Pakistan Journal of Medical Science, 2005, 21, 278-280.

[6] Erden S., Bryukazturk S., Langer S., Yilmaz G., Palanduz T., Badur S. International Journal of Kuwait University Health Science, 2003, 12 (13), 184-188.

[7] Weiss E. C., Makary M. A., Wang T., Syin D., Pronovost P. J., Chang D. Annals ofSurgery, 2005241, 803-805.

[8] Andre F. Hepatitis B epidemiology in Asia, the Middle East and Africa. Vaccine, 200018s, 20-22.

[9] Uneke C. J., Ogbu P. U. I., Anyanwu G. I., Njoku M. Oand Idoko J. H. Memorias do Instituto Oswaldocruz Cruz Rio de Janerio, 2005, 100 (1), 13-16.
[10] Akani C. I., Ojule A. C., Opurum H. C., Ejilemele A. A. Nigerian Postgraduate Medical Journal, 2005, 12 (4), 266-270.

[11] Umolu P. I., Okoror L. E., Orhue P. African Journal of Health Sciences, 20055 (1), 55-58.

[12] Krugman S., Overby L. R., Mushahwar I. K., Ling M., Frosner G. G., Deinhardt F. New England Journal of Medicine, 1979, 300, 101-106.

[13] Rinker J. and Galambos J. T. Gastroenterology, 1981, 9, 97115.

[14] Kay A. and Zoulim F. Virus Research, 2007127 (2), 164-176.

[15] EPINET. Exposure prevention information network data reports. University of Virginia; international health care worker safety center. 1999, Available fromwww.med.virginia.edu/epinet. retrieved on 28/02/2013.

[16] Alikor E. A., Erhabor O. N. Nigerian Journal of Medicine, 200716 (3), 250- 251.

[17] Olubuyide I. O., Ola S. O., Aliyu B., Dosomu O. O, Arotiba J. T., Olaleye O. A. East African Medical Journal, 199774, 357361 .

[18] Odusanya O. O., Maurice F. B., Hoet B. Transactions of the Royal Society ofTropical Medicine and Hygiene, 2007101 (5), 465-468.

[19] Adoga M. P., Gyar S. D., Pechulano S., Bashayi OD., Emiasegen S. E., ZungivT., Iperepulo O. H., Agupugo C and Agwale S. M. Journal of Infection in Developing Countries, 20104 (6), 397-400.

[20] Pido B. and Kagimu M. African Journal of Health Science, 2005, 5 (2), 93-98.

[21] Ahmad I., JanMahsud M. A., Hussain J., Khan M. H., Khan H., Noman N., Rabi $F$ and UdDin S. Gomal Journal of Medical Sciences, 2010, 8 (2), 102-104.

[22] Al-ajlan A. EMHJ, 201117 (10), 759-762.

[23] Lule G. N., Okoth F., Ogutu E. O., Mwai S. J. (1989). East African Medical Journal 198966 (5), 315-318.

[24] Dutta S., Shivanda P. G., Chatterjee A. Indian Journal of Public Health, 1994, 34, 108-112. 